\title{
ORBITAL STABILITY OF HIGH INCLINATION ASTEROIDS
}

\author{
N. A. SOLOVAYA \\ Celestial Mechanics Department \\ Sternberg State Astronomical Institute \\ University Prospect 13, 119889 Moscow, Russia \\ AND \\ E. M. PITTICH \\ Astronomical Institute of the Slovak Academy of Sciences \\ Dúbravská cesta 9, 842 28 Bratislava, Slovak Republic
}

\begin{abstract}
.
The orbital evolutions of fictitious asteroids with high inclinations have been investigated. The selected initial orbits represent asteroids with movement, which corresponds to the conditions of the Tisserand invariant for $C=C\left(L_{1}\right)$ in the restricted three body problem. Initial eccentricities of the orbits cover the interval $0.0-0.4$, inclinations the interval $40-80^{\circ}$, and arguments of perihelion the interval $0-360^{\circ}$. The equations of motion of the asteroids were numerically integrated from the epoch March 25, 1991 forward within the interval of 20,000 years, using a dynamical model of the solar system consisting of all planets. The orbits of the model asteroids are stable at least during the investigated period.
\end{abstract}

\section{Introduction}

The analytical theory of secular perturbations of asteroids with high inclinations and eccentricities shows that the changes of these elements are limited within certain small domains (Kozai, 1962). The theory was built with one disturbing body, Jupiter, on a circular orbit. The calculations of Gerasimov and Solovaya (1989) have shown that the outer part of the asteroid belt does not contain asteroids with high inclinations. Their orbits are not Hill's stable in this region. Indeed, we do not know of asteroids from the Hilda group having high inclinations. 
In this paper we have investigated the evolution of the group of fictitious asteroids which are located near of the boundary of the Hill's stability region. This boundary in the restricted three body problem is limited by the Jacobian constant, which is equal to the constant for the interior stationary point $L_{1}$. The semi-major axes of the orbits of the model asteroids were selected such that for arbitrary inclinations and eccentricities the condition $C=C\left(L_{1}\right)$ is always fulfiled. The question is whether the orbits of these asteroids are stable when they move in the dynamical system consisting of more perturbating bodies than in the three body dynamical system.

\section{Model orbits}

We took a dynamical model of the solar system, consisting of all planets and massless fictitious asteroids. We traced the 20,000 years orbital evolution of these asteroids using the numerical integration program with the RA15 integrator (Everhart, 1985). This period represents approximately 1770 revolutions of Jupiter. The initial asteroid semi-major axes $a_{0}$ were calculated from the equation for the integral of the type of Jacobi when $C=C\left(L_{1}\right)$. In the Sun-centered siderical coordinates has the following form:

$$
C=\left(\frac{1}{2 a}+\sqrt{p} \cos i\right)+\frac{1}{2} \mu^{2}+\mu\left(\frac{2 K\left(\kappa_{2}\right)}{\pi \nu}-R \cos b \cos \left(l-l_{j}\right)\right)
$$

where $\nu^{2}=\left(\rho^{2}+3 \alpha^{2}\right)^{2}, \alpha=(1-\mu) e_{j}$, and $\kappa_{2}^{2}=\left(4 \alpha^{2}-\left(\rho^{2}+3 \alpha^{2}-\nu^{2}\right) / 2\right) / \nu^{2}$. We used a notation similar as Solovaya et al. (1992), where $R$ is the Sunasteroid distance, $\rho$ is the Jupiter-asteroid distance, $\mu=0.0009538$ is the ratio of the Jupiter's mass to the sum of the masses of Sun and Jupiter, $e_{j}$ is the eccentricity of Jupiter's orbit, $l$ and $b$ are the longitude and latitude of an asteroid, $l_{j}$ is the longitude of Jupiter, $a, i$ and $p$ are the osculating elements and the parameter of an asteroid orbit, and $K\left(\kappa_{2}\right)$ is the elliptic integral. When $e_{j}=0$ then $K\left(\kappa_{2}\right)=\pi / 2$.

For the eccentricity of Jupiter $e_{j}=0.062$ the integral of the type of Jacobi $C=1.538$. The equation (1) was solved for $i_{0}$ equal $40^{\circ}, 60^{\circ}$, and $80^{\circ}$, and for $e_{0}$ equal $0.0,0.2$, and 0.4 . The initial value of argument of perihelion $\omega_{0}$ of the asteroids was selected from the equation $\cos \Theta_{\pi}=$ $\cos \omega \cos l_{j_{\pi}}+\sin \omega \sin l_{j_{\pi}} \cos i$ at $\Theta_{\pi}=180^{\circ}$ and varied with the step of $60^{\circ}$ from $0^{\circ}$ to $360^{\circ}$. The initial value of the ascending node $\Omega_{0}=90^{\circ}$. The initial epoch of the numerical integration was March 25, 1991 UT. For this moment $l_{j}$ was taken from Abalakin (1989). The extreme values of some orbital elements obtained from their evolution within the investigated period of the groups of asteroids with initial $\omega_{0} \in\left\langle 0^{\circ}, 360^{\circ}\right\rangle$ for initial values of $i_{0} \in\left\langle 40^{\circ}, 80^{\circ}\right\rangle$ and $e_{0} \in\langle 0.0,0.4\rangle$ are given in Table 1 . The evolution of the osculating eccentricity $e$, inclination $i$, perihelion disatance $q$ and 
aphelion distance $Q$ of the model asteroids with the initial inclinations $40^{\circ}$ and $80^{\circ}$ is plotted on Figure 1 and 2.

\section{Results of orbital integration}

The 20,000 years orbital evolution of the model asteroids shows a general behaviour of individual orbital elements in dependence on the initial $i_{0}$ and $e_{0}$. Within the investigated period all orbital elements of the model asteroids, except of $a$, change more or less periodically. The changes of $a$ are in all cases negligible. It means that all studied orbits have been stable at least for 20,000 years. The model orbits have more or less smaller $i$ after some time than at the beginning of the investigated evolution.

In the case of $i_{0}=40^{\circ}$ and $e_{0}=0.2$, some $i$ decline below $30^{\circ}$ after seven millenia, or when $e_{0}=0.4$ after four milenia. These changes repeat in periods of $\sim 14$ milenia in the first case, and $\sim 7$ milenia in the second one. For $e_{0}=0$, there is also the indication of the periodicity of the inclination change, but it must be more than 20,000 years. The evolution of $i$ of the asteroids with $i_{0}>40^{\circ}$ has similar behaviour except that the changes are relatively higher than in the previous case. The inclination decreased close to $30^{\circ}$ (see Figures and Table 1) for all investigated $i_{0}$.

The longitudes of perihelia of all model orbits change slowly. After some milenia the orbits with $e_{0}=0$ become similar for all initial arguments of perihelia, and there is no spread anymore. Within the investigated period, $e$ vary for all $i_{0}$ and $e_{0}$. The changes develop similarly as those of $i$ (see Figures and Table 1). The eccentricity of some orbits increased. Generally, the increase of $e$ is higher for higher $i_{0}$ and $e_{0}$.

These model asteroids is not only the change in the shape of their orbits, but also a movement of their orbits relative to the Sun. Of asteroids with $i_{0}=40^{\circ}$, only some with $e_{0}=0.4$ enter the region between the Sun and the Earth. But practically all asteroids with $i_{0} \geq 60^{\circ}$ enter this region during the 20,000 years. The exceptions are those with $e_{0}=0^{\circ}$. The perihelion distance of the asteroids reaches $0.68 \mathrm{AU}$ for $i_{0}=40^{\circ}$ and $e_{0}=0.4$. For $e_{0} \geq 0.2$ and $i_{0}=40^{\circ}$ the asteroids approach the Sun to the distance of $0.42 \mathrm{AU}$ and in the case of $i_{0}=80^{\circ}$ to $0.04 \mathrm{AU}$. Generally, the values of $q$ are smaller for higher $i_{0}$ and $e_{0}$ (see Figures and Table 1).

A group of the investigated model asteroids moves a little further from Jupiter. The aphelion distance of these asteroids with the initial inclination $40^{\circ}$ is not higher than $4.26 \mathrm{AU}$, with $60^{\circ} \leq 3.82 \mathrm{AU}$, and with $80^{\circ} \leq$ $3.58 \mathrm{AU}$. Therefore, and with regard to high inclinations, the motion of the investigated asteroids is disturbed with the Earth type planets.

Some of these asteroids are delivered from the main asteroid belt to the near-Mars space and then to the near-Earth space. These orbital changes 

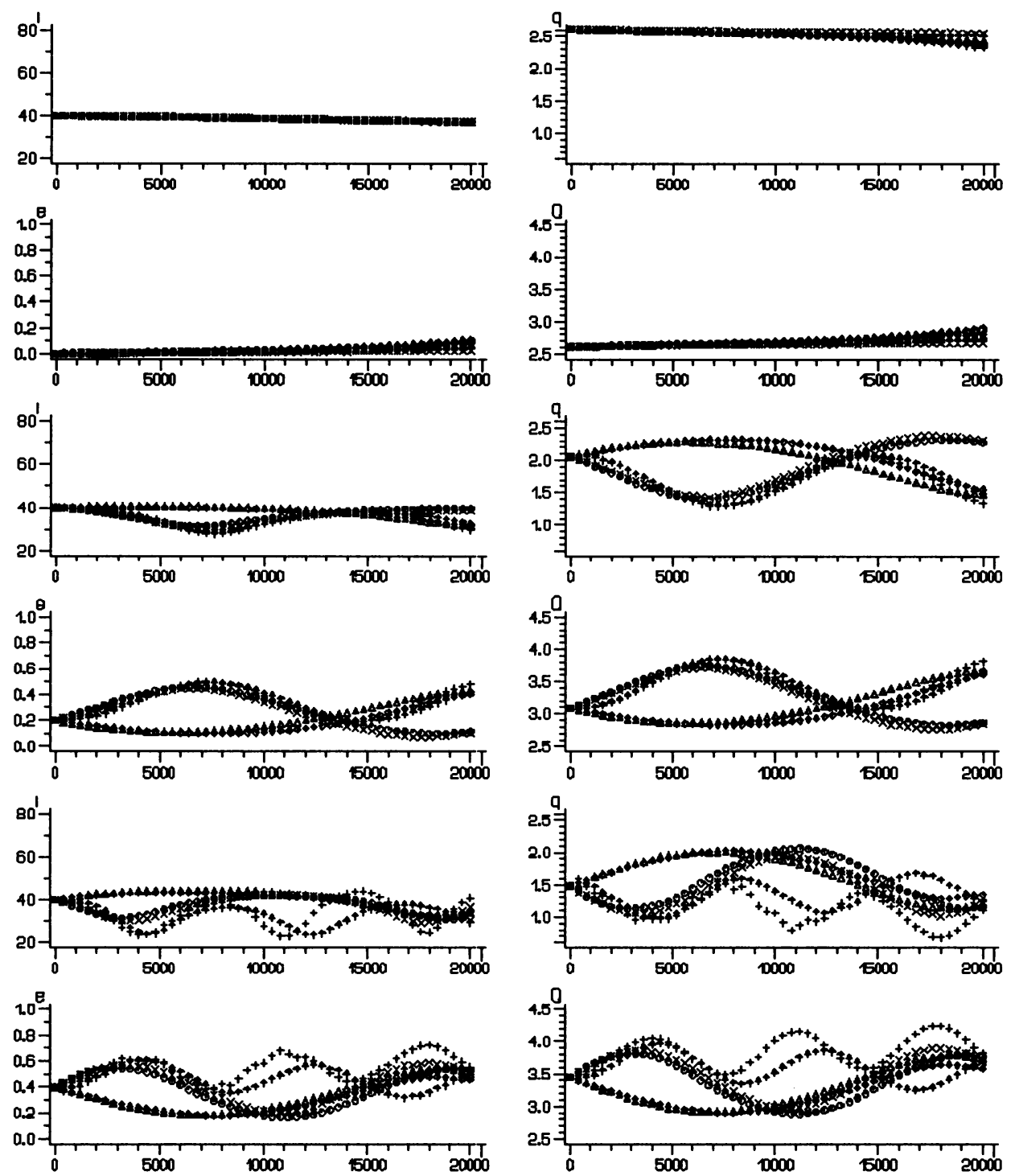

Figure 1. Evolution of orbital elements of the model asteroids within the period of 20,000 years. The initial asteroid inclination $i_{0}=40^{\circ}$, the eccentricity $e_{0}=0.0$ (top), $e_{0}=0.2$ (middle), and $e_{0}=0.4$ (bottom), and the longitude of perihelion $\omega_{0}: \odot-\omega_{0}=0^{\circ}, \Delta-$ $\omega_{0}=60^{\circ},+-\omega_{0}=120^{\circ}, x-\omega_{0}=180^{\circ}, \diamond-\omega_{0}=240^{\circ}$, and $4-\omega_{0}=300^{\circ}$.

are more frequent for higher $i_{0}$ and $e_{0}$ than for lower ones. They are not much depended on $\omega_{0}$. Thus, of the asteroids with $i_{0}=40^{\circ}$, about $20 \%$ might be delivered to the near-Earth region, of those with $i_{0}=60^{\circ}$ about $50 \%$, and of the asteroids with $i_{0}=80^{\circ}$ about $90 \%$ are delivered to this region within twenty millenia. 

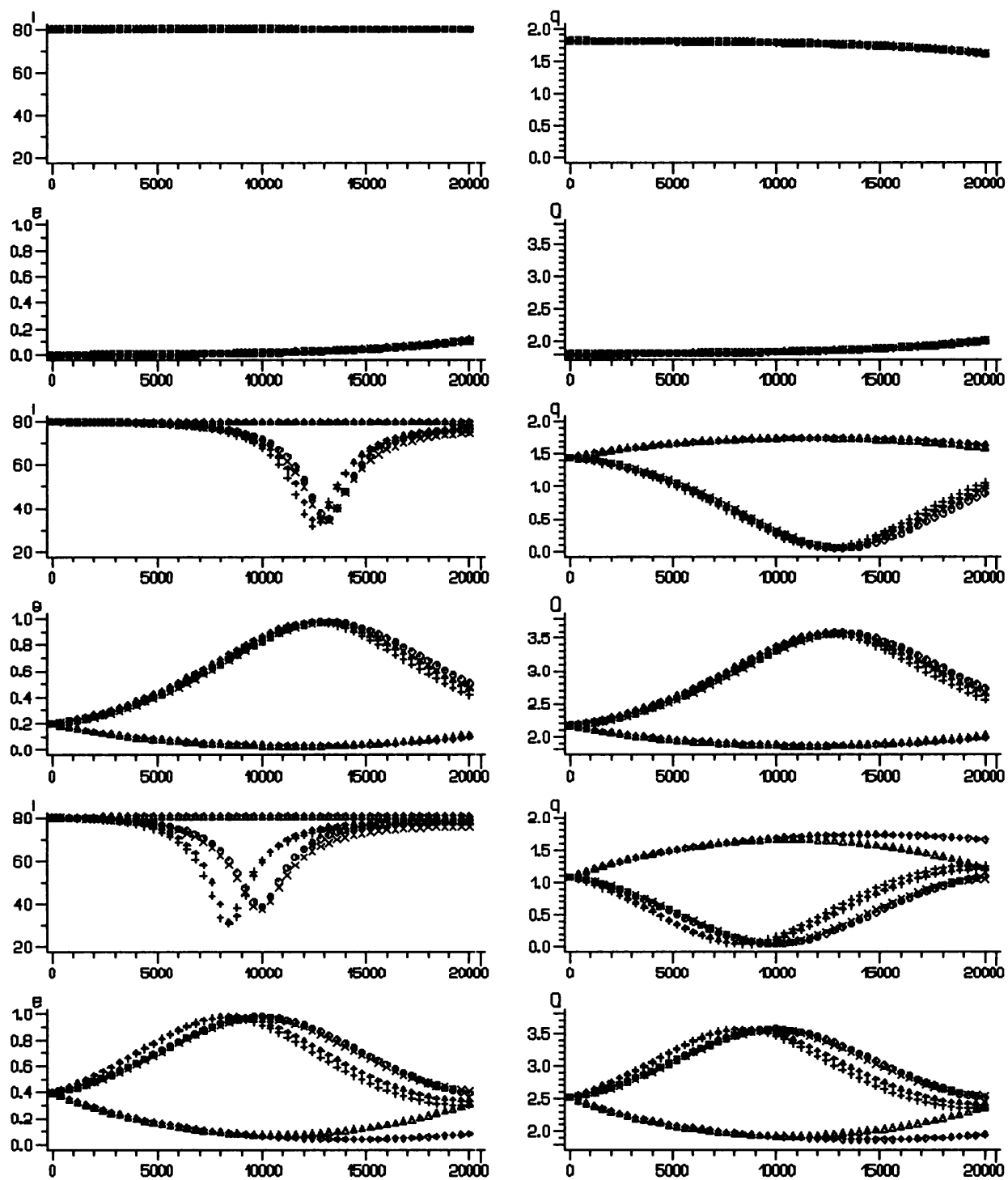

Figure 2. Evolution of orbital elements of the model asteroids within the period of 20,000 years. The initial asteroid inclination $i_{0}=80^{\circ}$, the eccentricity $e_{0}=0.0$ (top), $e_{0}=0.2$ (middle), and $e_{0}=0.4$ (bottom), and the longitude of perihelion $\omega_{0}: 0-\omega_{0}=0^{\circ}, \Delta-$ $\omega_{0}=60^{\circ},+-\omega_{0}=120^{\circ}, x-\omega_{0}=180^{\circ}, \diamond-\omega_{0}=240^{\circ}$, and $\uparrow-\omega_{0}=300^{\circ}$.

\section{Conclusion}

The behaviour of asteroidal orbits with high inclinations were considered in 20000 years interval and presented in plots. This time span is small for the discovery of secular resonances but sufficient for the investigation of the Hill's stability. The initial semi-major axes of the asteroidal orbits were 
TABLE 1. Time evolution of orbital elements of the model asteroids.

\begin{tabular}{ccccccc}
\hline $\mathrm{i}_{0}[0]$ & $\mathrm{e}_{0}$ & $\mathrm{i}^{[0]}$ & $\mathrm{e}$ & $\mathrm{a}[\mathrm{AU}]$ & $\mathrm{q}[\mathrm{AU}]$ & $\mathrm{Q}[\mathrm{AU}]$ \\
\hline 40 & 0.0 & $37-40$ & $0.00-0.11$ & $2.611-2.613$ & $2.32-2.61$ & $2.61-2.91$ \\
& 0.2 & $28-40$ & $0.07-0.49$ & $2.568-2.579$ & $1.30-2.39$ & $2.76-3.81$ \\
& 0.4 & $24-44$ & $0.18-0.70$ & $2.455-2.538$ & $0.68-2.02$ & $2.90-4.26$ \\
60 & 0.0 & $57-60$ & $0.00-0.32$ & $2.135-2.137$ & $1.46-2.14$ & $2.14-2.82$ \\
& 0.2 & $32-60$ & $0.12-0.81$ & $2.113-2.124$ & $0.43-1.86$ & $2.38-3.82$ \\
& 0.4 & $28-62$ & $0.25-0.86$ & $2.052-2.092$ & $0.42-1.56$ & $2.61-3.82$ \\
80 & 0.0 & 80 & $0.00-0.12$ & $1.812-1.812$ & $1.60-1.81$ & $1.81-2.02$ \\
& 0.2 & $33-80$ & $0.03-0.96$ & $1.806-1.812$ & $0.04-1.76$ & $1.86-3.58$ \\
& 0.4 & $31-81$ & $0.40-0.98$ & $1.799-1.805$ & $0.04-1.73$ & $1.87-3.56$ \\
\hline
\end{tabular}

taken so that the asteroids with $e_{0}$ and $i_{0}$ were located at the boundary of the Jacobi's sphera in the restricted three body problem. The chosen maximum value of $e_{j}$ makes this region smaller. It is interesting to follow the behaviour of the asteroids when the perturbations of all planets are taken into account. The Hill's stability or unstability of an asteroid could be found already at the order of 50 revolutions of Jupiter. The results of integration showed, that $e$ and $i$ change fluently, without large jumping and these changes are limited. The asteroids may be delivered to the near-Mars and to the near-Earth region, but after some period will be returned to the initial belt if the phenomena of resonanses will have not take place.

\section{Acknowledgements}

This work was supported by the Slovak Academy of Sciences Grant No. 2/1050/1995 (E. M. Pittich).

\section{References}

Abalakin, V.K. (1989) Astronomicheskij Ezhegodnik na 1991 god. Nauka, Leningrad.

Everhart, E. (1985) An efficient integrator that used Gauss-Radau spacing, Dynamics of Comets: Their Origin and Evolution, (A. Carusi and G.B. Valsecchi, Eds.), pp. 185202, Reidel, Dordrecht.

Gerasimov, I.A. and Solovaya, N.A. (1989) Evolution of the resonant group of minor planets of the outer part of the asteroidal belt, Asteroids Comets Meteors III, (C.-I. Lagerkvist, H. Rickman, B.A. Linblad, and M. Lindgren, Eds.), pp. 91-94, Uppsala University, Uppsala.

Kozai, Y. (1962) Secular perturbations of asteroids with high inclination and eccentricity, Astronomical Journal, Vol. no. 67, pp. 591-598.

Solovaya, N.A., Gerasimov, I.A., and Pittich, E.M. (1992) 3-D Orbital Evolution Model of Outer Asteroid Belt, Asteroids, Comets, Meteors 1991, (A.W. Harris and E. Bowell, Eds.), pp. 565-568, Lunar and Planetary Institute, Houston, Texas. 